\title{
Chapter 5 \\ AN INSTRUMENTATION SYSTEM FOR WAVE MEASUREMENTS, RECORDING AND ANALYSIS
}

\author{
by \\ H. G. Farmer and D. D. Ketchum \\ Woods Hole Oceanographic Institution \\ Woods Hole, Massachuset ts
}

\begin{abstract}
For the instrumentation system to be described, it was required that the system detect the sea surface accurately, be flexible in dynamic range, be able to detect and record at least six wave records simultaneously, be able to record the data at a station remote from the detectors, and be able to convert the data from analogue to digital form for analysis by electronic computers. Two types of wave measurements were required, the wave elevation and the wave slope. Resistance wire detectors were used and the theory of their operation is presented. The data acquisition and reduction system utilize recently developed telemetry techniques. Raw data storage is on magnetic tape using an inexpensive tape recorder and the digital data storage utilizes punched paper tape. The resistance wires have proven most satisfactory for small and large waves. The data acquisition and reduction system is sufficiently general that it should have application to other type investigations.
\end{abstract}

\section{INTRODUCTION}

The instrumentation system to be described in this paper was developed for use with several research projects concerned with wind generated waves. It was developed at the Woods Hole Oceanographic Institution (WHOI) and has been in operation for the last year. These investigations required that the wave elevation be measured at several discrete points, these several channels of data to be recorded simultaneously as a function of time. A closely allied experiment required measurement of the instantaneous slope of the sea surface referred to two horizontal axes aligned parallel and perpendicular to the wind direction. To accomplish these aims it was necessary to develop a suitable measuring instrument, and because of the expected large quantity of data and extensive calculations to be done, an efficient and practical means for recording and data reduction was needed. It was as sumed at the outset that nearly all of the calculations would be carried out on an electronic digital computer.

It was planned that a large number of the wave observations would be taken from a tower situated in Buzzards Bay, a line of sight distance of two miles from the Oceanographic Institution in Woods Hole.

*Contribution No. 1131 from the Woods Hole Oceanographic Institution. 


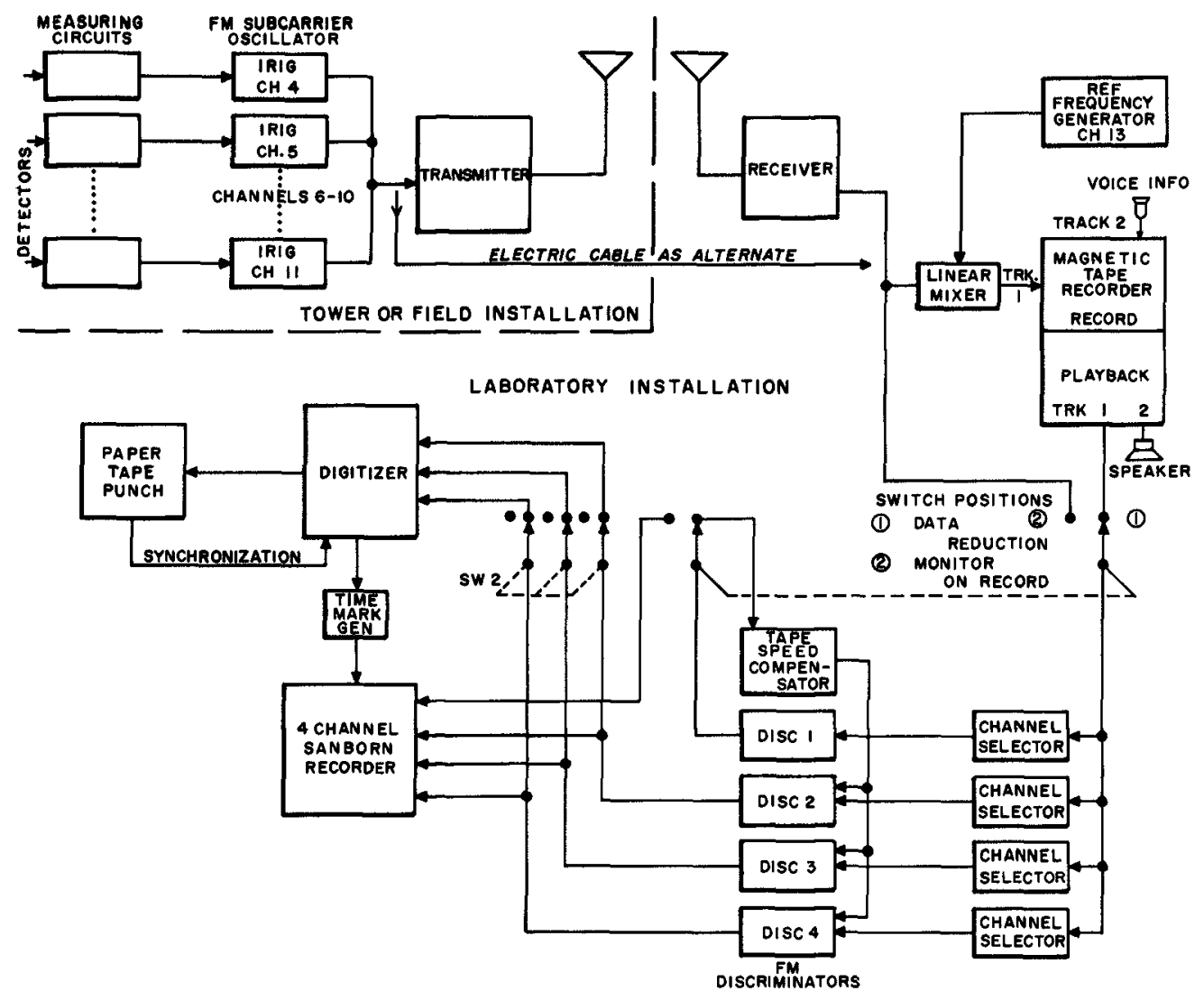

Fig. 1. WHOI multi-channel data acquisition and reduction system.
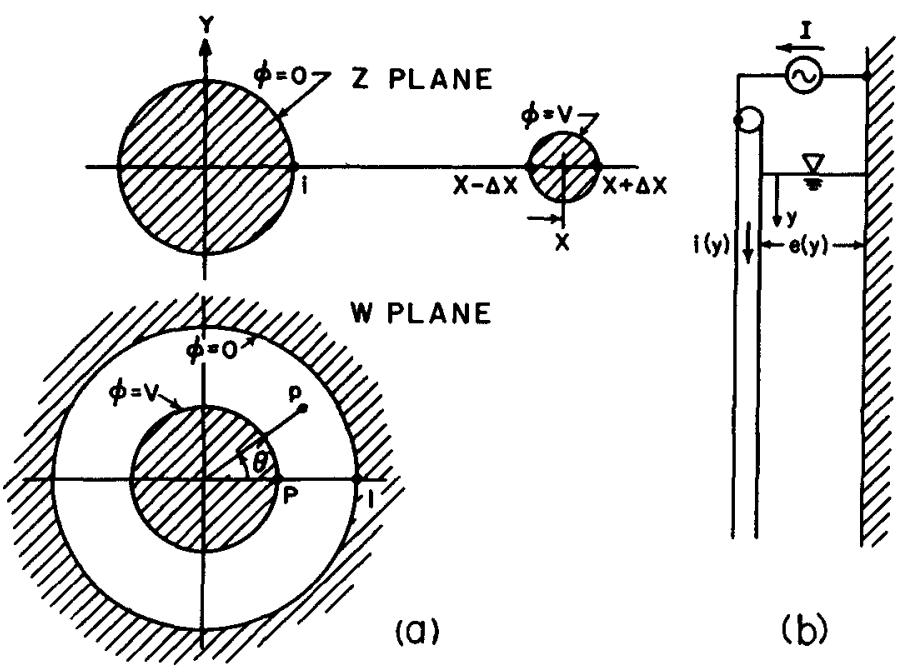

(b)

Fig. 2. (a) Geometry of wire and ground conductor and their transformation, (b) Schematic diagram of single wire transmission line and parallel ground plane, constant current source. 


\section{AN INSTRUMENTATION SYSTEM FOR WAVE MEASUREMENTS, RECORDING AND ANALYSIS}

Because of the physical conditions at the tower, only the necessary measuring circuits would be placed there and the several data channels, a total of eight, would be telemetered by radio to the laboratory, where they could be monitored and recorded. Furthermore, it was anticipated that observations would be made from other installations, and therefore the measuring and monitoring equipment should be kept portable.

An accurate measurement of the sea surface elevation immediately precluded any use of the well known pressure type wave recorder. Other techniques considered included the step-resistor type, Caldwel1 (1948), capacitance wire type, Tucker and Charnock (1955) and Whittenbury (1956) and the resistance wire type, Gerhardt, Jehn and Katz (1955). To take the difference in elevation of the sea surface between two points spaced close together, say approximately 6 inches, was considered adequate for the slope determination. This would require an accurate measure of the elevation at each point, and consequently the detectors must cause an absolute minimum disturbance to the water surface. It was hoped to make such measurements on waves up to six feet in height. For just the elevation observations, waves considerably higher than this were to be measured. The step-resistor gage was discarded as not having sufficient resolution, and the capacitance gage, although it was briefly investigated, was discarded as not being sufficiently reliable. The resistance wire gage had also been reported by Caldwell (1948), but was not rated favorably as a general purpose, durable, service instrument. The work of Gerhardt, Jehn, and Katz (1955), and also Clayton, Ivey and Teegardin (1954), suggested that this type of instrument, if properly designed and used, would be most satisfactory. A detailed analysis of this method of measurement was made for both the elevation and slope observations. An account of this analysis is given in a 1 ater section of this paper.

The complete multi-channel data acquisition and reduction system is illustrated in Fig. 1. In its most recent usage, the eight channels of data comprised six wave records, wind velocity and direction. With the provision of suitable measuring circuits there is no limit to the type of data that can be handled, except for the upper limit of the frequency content of the information. The system can easily accommodate up to twelve data channels, and, by eliminating the voice information, twenty-four data channels can be recorded. Data with frequencies up to $110 \mathrm{cps}$ can be faithfully recorded. A maximum frequency limit of about $10 \mathrm{cps}$ is imposed if the data is to be converted from analogue to digital form using the WHOI electronic digitizer. A $1 \%$ accuracy for the overall system was a desirable requirement. Rather than specify this, however, each component was selected for a $1 \%$ accuracy, or as close to this as practicable, so that the complete system might well be expected to have an accuracy of $3 \%$ Data would be recorded for time intervals up to one half an hour. This system will also be discussed in detail. The Inter-Range Instrumentation Group (IRIG) Standards used in the system are briefly described in the text. 
Finally, a brief description will be given of the field installation, a few practical considerations concerned with it and several examples of the type data collected.

Aside from the contribution this paper may make to the rather specialized subject of wave measurements, it is hoped that the telemetering and recording system will be of general interest since it is flexible enough to lend itself to a number of other uses without major modification. Although the techniques and most of the components involved are familiar to, and indeed were evolved largely be engineers in the missile industry, the authors are of the opinion that the subject is still sufficiently novel to oceanographers to warrant giving a description.

\section{THE RESISTANCE WIRE WAVE MEASURING SYSTEM}

The successful use of resistance wires for the measurement of waves requires careful design of the measuring circuits and a knowledge of the electrical behavior of the wires in the water. The wave height sensing devices consist of vertically supported stainless steel wires partly immersed in sea water. A constant current at audio frequency $(4 \mathrm{kc} / \mathrm{s})$ flowing in each wire and returning through the water path causes a voltage drop across the exposed portion of the wire proportional to the length of wire exposed. The voltage at the wire terminal after suitable amplification and detection is converted to a proper signal for telemetering to the laboratory for recording.

For the two types of measurements to be made, i.e., surface elevation and surface slope, the principal factors to be considered in the design are as follows:

a) The current and voltage distribution along the wire as a function of depth below the water surface;

b) The degree to which the generator feeding the wires must approximate an ideal current source;

c) The effect on the slope measuring circuits of the finite input impedance of the differential amplifiers;

d) The resolution of the wire in terms of the smallest measurable increment of the height of the surface.

In actual conditions of usage the resistance wire may be supported from quite different types of towers. At Woods Hole the wires were simply hung outboard of a light steel frame tower which served as the return path for the electrical current exciting the wire. This comprises 


\section{AN INSTRUMENTATION SYSTEM FOR WAVE MEASUREMENTS, RECORDING AND ANALYSIS}

a most complicated geometry to analyze in detail. Thus an analysis of (a) may best be performed by idealizing the geometry of the complete wire system. If the wire is considered to be supported parallel to a large vertical cylindrical conductor, partially immersed in a body of water of infinite extent, then it is possible to treat the immersed portion of the system as a transmission line with series resistance per unit length determined by the type of wire and shunt conductance per unit length determined by the geometry, the conductivity of the water and the polarization impedance at the wire-water interface.

In the following analysis, for the type of operation of the wire contemplated, it is found that for proper linearity of the voltage output with a constant current input it is necessary to maintain a certain minimum length of wire beneath the water surface at all times. For use in sea water with practical wire diameter and assumed geometry, the requirement for this minimum length of submerged wire may be easily met. This particular mode of operation of the wires proves impractical for use in fresh water. However, it is shown that a constant voltage system using a wire of very high conductivity should prove satisfactory. Further, for either the constant voltage or constant current system, it is shown that so long as certain minimum requirements are met, the exact current and voltage distribution along the subsurface portion of the wire are unimportant.

Since the specific resistance of the wire is known or can readily be measured, determination of the properties of the "transmission line" depends upon a knowledge of the shunt admittance existing between the wire and the ground plane. This admittance has two components in series: the polarization admittance arising from the electrochemical reaction at the surface of the wire electrode, and the admittance of the electro1yte, dependent upon the configuration of the electrodes and the physical properties of the water. Each of these admittances is complex, having a component of capacitive susceptance as well as conductance. However, it is well known to those famlliar with the technique of sea water conductivity measurements that at frequencies above about $1 \mathrm{kc} / \mathrm{s}$ the effect of the capacitance becomes small enough to neglect, and the polarization admittance is essentially constant at a fixed frequency. It is for this reason that the $4 \mathrm{kc} / \mathrm{s}$ frequency was selected for the wire excitation. Therefore, although the polarization admlttance accounts for a substantial part of the total effect, it can be regarded as a constant, which, since it is in series with the electrolyte admittance, reduces the total admittance to a value below that of the electrolyte admittance alone. In the case of the electrolyte admittance it is also true that the capacitance between the wire and the ground plane is small enough to be neglected at the operating frequency. Hence it only remains to calculate the real part of the electrolyte admittance, which will hereafter be simply termed the shunt conductance. 


\section{THE WIRE-GROUND CONDUCTOR FIELD PROBLEM}

The determination of the shunt conductance requires the solution of the field problem associated with the configuration shown in Fig. 1a, where a wire of radius $\Delta x$ is maintained at a potential $V$ with respect to a parallel conductor of unit radius. If it is assumed that the immersed part of the system extends to infinite depth, then because of symmetry the solution for the potential may be found in two dimensions. Application of the following transformation, churchill (1948), results in a coaxial configuration whose potential can easily be found:

$$
w=\frac{z-a}{a z-1}
$$

where

$$
a=\frac{1+(x+\Delta x)(x-\Delta x)+\sqrt{\left[(x+\Delta x)^{2}-1\right]\left[(x-\Delta x)^{2}-1\right]}}{2 x}
$$

and the transformed radius is

$$
\begin{aligned}
& \mathrm{P}=\frac{(\mathrm{x}+\Delta \mathrm{x})(\mathrm{x}-\Delta \mathrm{x})-1-\sqrt{\left[(\mathrm{x}+\Delta \mathrm{x})^{2}-1\right]\left[(\mathrm{x}-\Delta \mathrm{x})^{2}-1\right]}}{2 \mathrm{x}} \\
& \mathrm{x}-\Delta \mathrm{x}<\mathrm{a}<\mathrm{x}+\Delta \mathrm{x} \quad 0<\mathrm{P}<1 \text { when } \mathrm{x}-\Delta \mathrm{x}<1
\end{aligned}
$$

The potential $\phi$ of the transformed system is

$$
\phi=\frac{V}{\ln p} \ln p
$$

and the total current per unit length flowing from the wire to the large conductor may be found as follows :

$$
\begin{aligned}
& \overrightarrow{\mathrm{E}}=\operatorname{GRAD} \phi=-\frac{o \phi}{\partial_{\mathrm{p}}} \\
& i=-\sigma \int_{b}^{2 \pi} \quad \overrightarrow{\mathrm{E}} \mathrm{d} \theta=-\frac{2 \pi \sigma \mathrm{V}}{\ln \mathrm{P}}
\end{aligned}
$$

where $\sigma=$ the conductivity of the water $\vec{E}=$ the electric field vector

The shunt conductance per unit length is then

$$
g=\frac{i}{V}=-\frac{2 \pi \sigma}{\ln P}
$$




\section{AN INSTRUMENTATION SYSTEM FOR WAVE MEASUREMENTS, RECORDING AND ANALYSIS}

In the practical case being considered the wire diameter is very small compared with the radius of the ground conductor, making the accurate computation of $P$ tedious. The expression for $P$, eq. (3), may be simplified by replacing the square root term by the first two terms of its binominal expansion and neglecting the term in $\Delta x$ to the fourth power. This approximation, eq. (8), is therefore valid for values of $\Delta x<<x$.

$$
P=\Delta x\left(\frac{1}{x^{2}-1}\right)
$$

Rescaling the dimensions of the $\mathrm{Z} \mathrm{pl}$ ane to the actual physical dimensions

$$
P=\frac{k}{K} \quad\left(\frac{1}{D^{2} / k^{2}-1}\right)
$$

and from eq. (7) the shunt conductance becomes

$$
g=\frac{2 \pi \sigma}{\ln \frac{K}{k}\left(\frac{D^{2}}{k^{2}}-1\right)}
$$

where $\mathrm{D}=$ Distance between centers

$$
\begin{aligned}
& \mathrm{K}=\text { Ground conductor radius } \\
& \mathrm{k}=\text { Wire radius }
\end{aligned}
$$

RESISTANCE WIRE ANALYSIS, CONSTANT CURRENT SOURCE

With the value of the shunt conductance established, the wire and ground conductor may now be regarded as a single wire transmission line parallel to a perfectly conducting ground plane (Fig. 2b). This last assumption is valid since the actual ground conductor is a pipe whose series resistance, because of its relatively great diameter, is negligible compared to the series resistance of the wire. If the original assumption of infinite immersed depth is for the moment maintained, the classic transmission line equations may be written, without, however, the complication of accounting for the reactive components, since the frequency of the wire current will be low enough to neglect series inductance, and the leakage, or shunt conductance, is so high in sea water that the effect of shunt capacitance will be negligible.

The general solution for the voltage with respect to the ground plane and the current in the wire as functions of distance along the 


\section{COASTAL ENGINEERING}

wire are found by well-known methods, Guillemin, (1935), to be

$$
\begin{aligned}
& e(y)=A e^{\sqrt{\rho} \bar{g} y}+B e^{-\sqrt{\rho \bar{g}} y} \\
& i(y)=\sqrt{g} \bar{\rho}\left[-A e^{\sqrt{\rho \bar{g}} y}+B e^{-\sqrt{\rho \bar{g}} y}\right],
\end{aligned}
$$

where $\rho$ is the resistance per unit length of the wire, and $y$ is distance measured from water surface.

The constants are evaluated by applying the end conditions:

$$
i(0)=1, \quad i(\infty)=0
$$

where $I=$ the current in the wire above the water surface.

The voltage and current along the wire are seen to be simple exponential functions of depth.

$$
\begin{aligned}
& i=I e^{-\sqrt{\rho_{g}} y} \\
& e=I \sqrt{\rho / g} e^{-\sqrt{\rho_{g}} y}
\end{aligned}
$$

of interest is the depth at which the current decays to a negligible value. If this critical depth $y_{c}$ is defined as the depth at which the current in the wire is reduced to $1 \%$ of $\mathrm{I}$, then

$$
y_{c}=\frac{4.60}{\sqrt{\rho g}}
$$

It therefore follows that so long as the wire is immersed at least to its critical depth, it will behave as though immersed to infinite depth, and the voltage drop from the wire to ground at the water surface will remain constant as the level of the water changes with respect to the wire. It is this fact that permits us to regard the instantaneous level of the water surface as the position of a short circuit to ground, since the constant voltage drop simply represents a constant zero shift which can be calibrated out.

Substituting the formula for the shunt conductance into eq. (16) and expressing $\rho$ in terms of the wire radius shows how the critical depth depends upon the wire radius with all other parameters held constant. The result of this manipulation is

$$
y_{c}=\frac{4.60 k}{\sqrt{2 \sigma \mu^{\prime}}} \quad \sqrt{\ln \left[\frac{K}{k}\left(\frac{D^{2}}{K^{2}}-1\right)\right]}
$$




\section{AN INSTRUMENTATION SYSTEM FOR WAVE \\ MEASUREMENTS, RECORDING AND ANALYSIS}

where $\rho^{\prime}$ is the resistivity of the metal used for the wire. The critical depth is found to be very nearly linear with respect to the wire radius as is seen in Fig. 3. This is a plot of eq. (17) for the case of stainless steel wire spaced 10 inches on centers from a pipe of one inch radius in sea water of conductivity 0.127 mhos per inch.

When the wire is immersed to a depth $\lambda$ which is less than the critical depth, the behavior of current and voltage along the wire as a function of depth $c$ an be found by noting that the end conditions now become

$$
i(0)=I, i(\lambda)=0
$$

Thus :

$$
\begin{aligned}
& i(y)=I \frac{\sinh \sqrt{\rho_{g}^{-}}(\lambda-y)}{\sinh \sqrt{\rho_{g}^{-}} \lambda} \\
& e(y)=I \sqrt{\rho / g} \frac{\cosh \sqrt{\rho_{g}^{-}}(\lambda-y)}{\sinh \sqrt{\rho_{g}^{-}} \lambda}
\end{aligned}
$$

Under these conditions the voltage at the water surface and the vol tage at the end become respectively

$$
\begin{aligned}
& \text { e }(0)=I \sqrt{\rho / g} \quad \operatorname{coth} \sqrt{\rho_{g}} \lambda \\
& \text { e }(L)=I \sqrt{\rho / g} \quad \operatorname{csch} \sqrt{\rho_{g}^{-}} \lambda
\end{aligned}
$$

The voltage at the surface was measured experimentally in a tank of sea water, using .015" stainless steel wire spaced 5.5 inches on centers from a 3/4" diameter stainless steel pipe. The curve from this experimental data was found to be similar in shape to eq. (22) but displaced from it. This discrepancy is expected to be due principally to the effect of the unknown but nonetheless real polarization admittance. For the manner in which the resistance wires were to be used an estimate of this polarization admittance was not required. However, an experiment of this sort could provide such an estimate.

In situations where it may be impractical to employ a wire of sufficient length to reach critical depth, terminating the wire in its characteristic impedance, $\sqrt{\mathrm{p}} / \mathrm{g}$, should, in theory, simulate an infinite length of wire below the surface. In this manner e (0) could be held constant for short lengths of wire.

In order for the voltage drop across the exposed portion of the wire to be proportional to the exposed length, the current I must be constant and independent of the position of the water surface. Indeed, 


\section{COASTAL ENGINEERING}
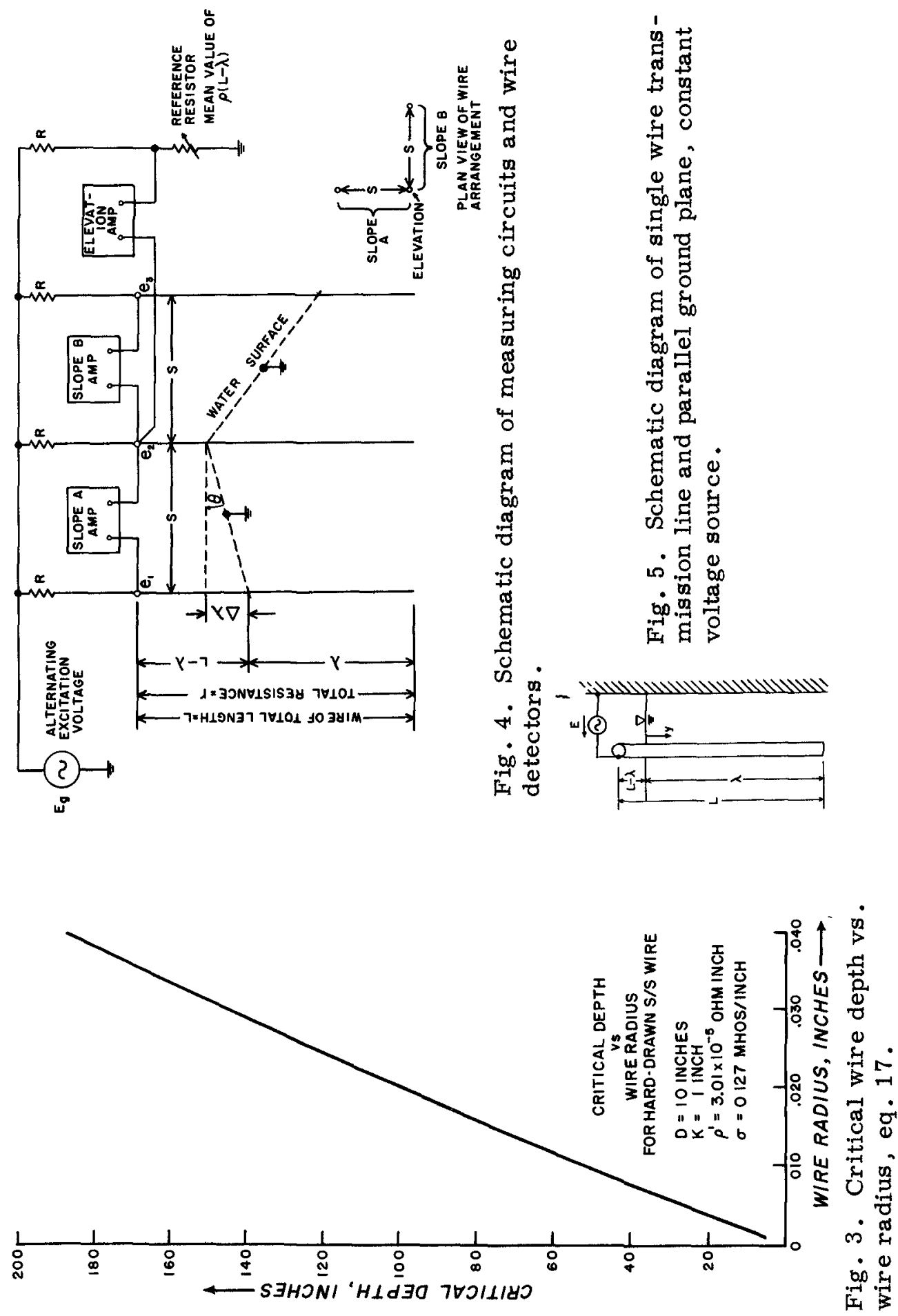


\section{AN INSTRUMENTATION SYSTEM FOR WAVE MEASUREMENTS, RECORDING AND ANALYSIS}

the preceding analysis is based upon this assumption. The accuracy of the device is therefore dependent, among other things, upon two factors: namely, the degree to which the wire excitation source approximates a current source, and the degree to which the input impedance of the amplifiers associated with the wire approximates an open circuit.

The diagram in Fig. 4 shows how the equal current sources are approximated. Current regulation with respect to load variations is achieved by making the resistors $R$ large compared to the sensing wire resistance $\mathrm{r}$. The voltage source $\mathrm{E}_{\mathrm{g}}$ is electronically regulated for stability. A simple calculation shows the ratio of series resistance to wire resistance required for a given percentage linearity of the output voltage appearing between one of the terminals and the water.

Assuming for the moment that the inputs of the amplifiers are open circuits, and for simplicity that the water surface is a short circuit, we will consider one individual wire. It is desired that the relation between the wire output voltage and the water height be linear within specified limits over the total range of interest. The output voltage is given by

$$
e_{2}(\lambda)=\frac{E_{g} r / L(L-\lambda)}{r / L(L-\lambda)+R}
$$

The elevation sensitivity $d e_{2} / d \lambda$ must remain between specified limits to insure the desired linearity.

Thus for $1 \%$ inearity,

$$
\left.\frac{d e_{2}}{d \lambda}\right|_{L}-\left.\frac{d e_{2}}{d \lambda}\right|_{0}=\left.\frac{1}{100} \quad \frac{d e_{2}}{d \lambda}\right|_{0}
$$

Solution of (25) for $R$ yields

$$
\mathrm{R}=200 \mathrm{r}
$$

In the case of a slope determination the difference between the voltages at the terminals of two parallel wires whose spacing is known is measured by means of a differential amplifier. Analysis of the effect of the ratio $R / \mathrm{r}$ on the accuracy of this measurement shows that the voltage representing the slope is not nearly so sensitive to change in the elevation of the water surface as the elevation linearity. If we consider a pair of wires and assign the symbols $e_{1}$ and $e_{2}$ to the voltages at the terminals of the two wires, see Fig. 4, with respect to 


\section{COASTAL ENGINEERING}

the water surface (considered as ground), then the slope of the water surface in the direction of a line connecting the two wires is proportional to $e_{12}$, the difference between $e_{1}$ and $e_{2}$. We must investigate how this difference varies for a constant slope over the length $\lambda$. Sufficient information for this purpose is given by comparing the expressions for the slope voltage at maximum and minimum elevation. Thus,

$$
e_{12}=e_{1}(\lambda)-e_{2}(\lambda+\Delta \lambda)
$$

and eq. (23) may be used for $e_{1}$ and $e_{2}$.

By taking the difference of $e_{12}$ determined at $\lambda=L-\Delta \lambda$ and $\lambda=0$, assuming $\mathrm{r} \Delta \lambda<<\mathrm{R} \mathrm{L}$, and allowing a maximum slope voltage variation of $1 \%$ over the range $L$, the following value of the resistance $R$ is obtained

$$
\mathrm{R}=1.58 \mathrm{r}
$$

Thus the requirement of eq. (26) more than fulfills the requirement for slope voltage accuracy over the length of the resistance wires.

The preceding discussion has been based on the assumption that the input impedance of the differential amplifiers is infinite. Although this is far from the actual case, it may be shown that if these impedances can be made high compared to the wire resistance, the above assumption is valid. Since the highest value of wire resistance contemplated in this design is about 20 ohms, it was possible to obtain an adequately high input impedance to the differential amplifiers which are of transistor design.

In the preceding analyses, the derived relations define the parametes of the sensing circuit and establish criteria for the sensitivity of the amplifier detector units and the output level of the stabilized voltage source. The maximum exposed wire resistance was chosen as nominally 20 ohms, which corresponds to about 10 feet of .015" diameter stainless steel wire. Thus, in providing the constant current source for the wire excitation, the series resistors $R$ must be at least 4000 ohms according to eq. (26). Precision resistors with an accuracy. $\pm .05 \%$ were used to insure that the currents flowing in each wire were identical.

\section{RESISTANCE WIRE ANALYSIS, CONSTANT VOLTAGE SOURCE}

An extension of the foregoing analysis shows how a wire may be used to measure waves in fresh water, where the conductivity is several orders of magnitude less than that of sea water. In this case a wire of very low specific resistance is driven with a voltage source $E$, as shown in 


\section{AN INSTRUMENTATION SYSTEM FOR WAVE \\ MEASUREMENTS, RECORDING AND ANALYSIS}

Fig. 5. Below the fresh water surface $y \geqq 0$ equations 11 and 12 hold, and for $\mathrm{y} \leqslant 0$ :

$$
\begin{aligned}
& e(y)=E-\rho(L-\lambda+y) i_{0} \\
& i(y)=i_{0}=i(0)
\end{aligned}
$$

Applying the end conditions

$$
\begin{aligned}
& \text { e }(0)=A+B=E-\rho(L-\lambda) i_{0} \\
& i(\lambda)=0=-A e^{\sqrt{P G} \lambda}+B e^{-\sqrt{\rho g} \cdot \lambda}
\end{aligned}
$$

and solving for $i_{0}$, we find the current in the exposed portion of the wire to be

$$
i_{0}=\frac{\left(1-e^{-2} \sqrt{\rho \bar{g}} \lambda\right) \sqrt{g / \rho} E}{e^{-2 \sqrt{\mu_{g}} \lambda}+1+\left(1-e^{-2 \sqrt{\rho g} \lambda}\right) \sqrt{\rho g}(L-\lambda)}
$$

So long as $2 \sqrt{\rho_{g}} \lambda<<1$, equation (33) reduces to the approximation

$$
i_{0}=g \mathrm{E} \lambda
$$

showing that the current from the voltage source is directly proportional to the length of the immersed portion of the wire. For the range of conductivity encountered in most tap water it appears that the above criterion can be met by using copper wire of moderate diameter in lengths up to 10 feet. Calculation of the shunt capacitance of a typical system indicates that at low to medium audio frequencies the shunt susceptance is very small compared to the shunt conductance in fresh water. Hence as in the sea water system the analysis is valid without consideration of the reactive components.

\section{VERIFICATION, MEASUREMENT RESOLUTION AND ADDITIONAL COMMENTS}

It was found experimentally that the mean resolution of the currentdriven wave measuring wire was limited to the order of 0.2 inch for a 12 foot length of wire. This result was demonstrated by an experiment where a pair of parallel wires was lowered and raised in a deep tank of sea water over a distance of 12 feet and maintained perpendicular to the calm surface. The output voltage from a single wire was measured for elevation. The output voltage from the differential amplifier fed from the two wire terminals, instead of remaining at zero, was observed to vary about its zero value in an erratic fashion. With a wire spacing of 6 inches the standard deviation from the mean value corresponded to 0.17 inch which was equivalent to a slope of $1.6^{\circ}$. Since careful attention had been devoted to the design of the differential amplifier, this effect could not be attributed to faulty common mode rejection or imperfect balance in the 


\section{COASTAL ENGINEERING}

electronic circuits. The elevation measurements over the full range of inmersion indicated the predicted linearity, but the difference measurements provided a measure of the resolution available.

The only plausible explanation for this "noise" appeared to be small random variations in the polarization admittance caused by surface contamination of the wire. Thus it was at least logical to assume that a thin but non-uniform 1 ayer of grease or contaminate could result in the admittance varying randomly with depth along the wire. Since the polarization admittance is a component of the shunt conductance, $g$, variations in the former would cause variations in e (0), upon whose constancy with surface height the system depends for its 1inearity. This thesis was partially supported by the fact that degreasing agents applied to the wires immediately prior to immersion caused changes in the effect, without, however, eliminating it.

In any case, since it would not be possible to maintain uncontaminated wires in field operation, a full-scale range of the order of two feet seems to be the practical limit where accuracy of one per cent of full scale is desired in an elevation wire, while the spacing of slope wires cannot be less than about six inches for angular accuracy of better than about $2^{\circ}$.

In the equipment as it was actually built the signals from each of the elevation wires as well as the signals from the pairs of slope wires were amplified with differential amplifiers and detected with phase sensitive detectors. By adjustment of the various gain settings, the final output voltage was standardized to a 0-5 volt range for entry into the telemetering equipment. Differential amplifiers were used in the elevation circuits so that it would be possible to subtract from the wave wire signal another signal equivalent approximately to the mean length of unimmersed wire, refer to Fig. 4. This reference resistor is variable so that a proper adjustment may be made whatever the state of the tide. The total "swing" of the differential amplifier output is then primarily due to just the wave signal. The amplifier sensitivity is adjusted so that its full scale excursion in both directions is caused by the highest expected waves. The variable reference resistor may take the form of another wave wire which is supported in a tide tube. This tube would be designed so that the reference wire senses only tidal variation.

The design of the differential amplifier, particularly those for the slope measurements, required great care to be taken to insure high common mode rejection. The common mode rejection ratio of the amplifiers is determined by measuring the differential gain, the ratio of output vol tage to the difference between the two input voltages. The two input voltages are made equal by tying the two input terminals together and the ratio of the output voltage to the common input voltage, or common mode gain, is measured. The common mode rejection ratio obtained was in the order of 10,000 . 


\section{AN INSTRUMENTATION SYSTEM FOR WAVE MEASUREMENTS, RECORDING AND ANALYSIS}

The $4 \mathrm{kc} / \mathrm{s}$ outputs of the differential amplifiers are detected in phase sensitive detectors, the magnitude of whose D.C. output is proportional to the amplified difference voltage applied. The polarity of each phase detector output is dependent upon the phase of the input compared to that of the fixed reference phase of the wire drive supply. Since the difference voltage undergoes a phase reversal at the point where the voltage difference input to the amplifier becomes zero, the sense of the instantaneous water level is preserved.

\section{WHOI MULTI CHANNEL DATA ACQUISITION AND REDUCTION SYSTEM}

The complete system in its present operational form is illustrated in Fig. 1. Two main divisions of the equipment have been made, the field installation and the laboratory installation. The means for telemetering the information between these two stations may be either by radio or electrical cable with each method requiring its own special terminal equipment. All of the data received at the laboratory in its full analogue form is recorded on magnetic tape, which serves as the raw data storage. Upon playback the data may be monitored, edited, digitized, or otherwise operated upon without altering the original data recording. Since the analysis was to be carried out on an electronic digital computer it was necessary to provide an analogue-todigital converter and a means of storage, punched paper tape, for the digital information.

Besides the accuracy requirement of $1 \%$ for each component it was considered important to keep the system as adaptable as possible to a wide variety of applications and problems. As always it was necessary to keep the overall cost at a minimum.

The design of the telemetering apparatus was fortunately much simplified by the fact that the art of FM/FM telemetry has reached the advanced state where standard components are available from commercial manufacturers. Standard subcarrier frequencies, channel spacings and bandwidths, designed to permit optimum use of the available spectrum consistent with the requirements of bandwidth and signal-to-noise ratio have for some years been promulgated by the Inter-Range Instrumentation Group (IRIG) and are widely accepted by the industry. Nichols and Rauch (1957) give a comprehensive introduction to the theory and practice in the field of radio telemetry. When analogue information is to be transmitted any distance by radio, it is well known that frequency modulation best preserves the information against distortion by extraneous noise. Similarly, for magnetic tape recording as well as for radio transmission when the information data frequencies extend down to zero it is necessary to utilize frequency modulation (FM) to preserve this information. To 


\section{COASTAL ENGINEERING}

obtain a number of data channels for transmission over a single radio frequency carrier or for recording on a single track magnetic tape recorder, the range of frequencies extending from about $.4 \mathrm{kc}$ to $70 \mathrm{kc}$ has been divided up into a number of bands each of which is assigned a center frequency. See Table $I$. The information signal is then used to frequency modulate this center frequency, the maximum deviation for each band being $\pm 71 / 2 \%$ of its center frequency. From the table it will be seen that the maximum intelligence frequency depends on the subcarrier band selected. The WHOI system utilizes the IRIG data channels 4 through 11 but can accommodate channels 1 through 12 .

A most satisfactory means for recording these several channels of data is on magnetic tape. It is significant to note that an expensive instrumentation recorder is not required. A relatively inexpensive, portable, hi-fidelity, dual track tape recorder has proven adequate for the job. One track is used for data recording, and voice information is put on the second track. In this manner the data may be documented with whatever additional information is needed while it is being recorded. Because of slight irregularities in the motion of the tape on both record and playback, occurring in all tape recorders, FM recorded data may be very seriously distorted. This difficulty can be virtually eliminated, however, by recording along with the data a stable reference or control frequency. Upon playback the variations in this frequency are used to correct the data restoring it to its original form well within the accuracy limits of the overall system. This correction process is referred to as tape speed compensation.

The field installation comprises the detectors, measuring circuits, FM subcarrier oscillators and, if required, a radio transmitter. The FM subcarrier oscillators are voltage controlled, a 0 to 5 volt swing at the input causing the output frequency to range from its lower to its upper limit. In order effectively to utilize the accuracy available in this telemetering and recording system, the sensitivity of the measuring circuits should be so adjusted that full scale of the information corresponds to the full $0-5$ volt range of the subcarrier oscillator. Generally this can be easily done. The outputs of the oscillators are then linearly mixed and the combined signal is fed to the FM transmitter. It is by virtue of the information frequency modulating the subcarriers, and the several subcarriers frequency modulating the.radio frequency carrier that the system is referred to as FM/ FM telemetry. In those field installations where electrical power is primarily supplied by batteries, it is desirable to use as much transistorized circuitry as possible in order to prolong battery life and reduce the number of batteries required. Transistorized subcarrier oscillators are commercially available and are extremely rugged and highly dependable. The measuring circuits designed and built at WHOI for the wave observations were also transistorized. 
AN INSTRUMENTATION SYSTEM FOR WAVE

MEASUREMENTS, RECORDING AND ANALYSIS

TABLE I

IRIG STANDARD SUBCARRIER BANDS

\begin{tabular}{|c|c|c|c|c|c|}
\hline & Center & & Lower & Upper & Maximum Intelligenc \\
\hline & $\begin{array}{l}\text { Frequency } \\
\quad \text { (cps) }\end{array}$ & $\begin{array}{l}\text { Deviation } \\
\text { (cps) }\end{array}$ & $\begin{array}{l}\text { Limit } \\
\text { (cps) }\end{array}$ & $\begin{array}{l}\text { Limit } \\
\text { (cps) }\end{array}$ & $\begin{array}{c}\text { Frequency } \\
\text { (Modulation Index } 5\end{array}$ \\
\hline
\end{tabular}

$\pm 71 / 2 \%$ Deviation Channels

$\begin{array}{rcrrrr}1 & 400 & \pm 30 & 370 & 430 & 6.0 \\ 2 & 560 & 42 & 518 & 602 & 8.4 \\ 3 & 730 & 55 & 675 & 785 & 11 \\ 4 & 960 & 72 & 888 & 1,032 & 14 \\ 5 & 1.3 \mathrm{kc} / \mathrm{s} & 98 & 1,202 & 1,398 & 20 \\ 6 & 1.7 & 128 & 1,572 & 1,828 & 25\end{array}$

\begin{tabular}{rcrrrr}
\hline 7 & 2.3 & 173 & 2,127 & 2,473 & 35 \\
8 & 3.0 & 225 & 2,775 & 3,225 & 45 \\
9 & 3.9 & 293 & 3,607 & 4,193 & 59 \\
10 & 5.4 & 405 & 4,995 & 5,805 & 81 \\
11 & 7.35 & 551 & 6,799 & 7,901 & 110 \\
12 & 10.5 & 788 & 9,712 & 11,288 & 160 \\
\hline & & & & & \\
13 & 14.5 & 1,088 & 13,412 & 15,588 & 220 \\
14 & 22.0 & 1,650 & 20,350 & 23,650 & 330 \\
15 & 30.0 & 2,250 & 27,750 & 32,250 & 450 \\
16 & 40.0 & 3,000 & 37,000 & 43,000 & 600 \\
17 & 52.5 & 3,940 & 48,560 & 56,440 & 790 \\
18 & 70.0 & 5,250 & 64,750 & 75,250 & 1,050 \\
\hline
\end{tabular}




\section{COASTAL ENGINEERING}

In choosing the means for telemetering the information from the field to the laboratory installation several factors must be considered. Among these are the total number of data channels, the channel center frequencies, the range of transmission, whether or not line of sight communication exists, etc. Generally if a large number of data channels are required and IRIG channel 7 or greater is used, the total band width of these data channels will require for radio transmission a VHF (say $230 \mathrm{mc}$ ) carrier with frequency modulation. This VHF-FM radio link will provide high quality telemetry, but the line of sight transmission may introduce a range limitation. With fewer data channels, IRIG 1 thru 6, the total band width of the several channels will permit use of an HF (2-6 mc) carrier but with amplitude modulation. In this case, the range of transmission will be greatly increased but there may also be some sacrifice in quality.

Electrical cables may also be used for the telemetering link. In this case the subcarrier signals are fed directly to the cable. Here again the band width and frequencies of the transmitted signals will dictate the selection of the cable. Generally if the band width is large and the higher frequency data channels are used a coaxial cable will be required.

At the laboratory the output of the FM receiver is 1inearly mixed with the reference frequency, $14.5 \mathrm{kc} / \mathrm{s}$ the center frequency of IRIG channel 13, and recorded with the magnetic tape recorder. At the same time, with switch 1 in position 2 any four of the data channels may be selected, using the appropriate channel selectors, sharp cut off band pass filters, discriminated and recorded on the four channel Sanborn recorder. This ability to monitor the data while it is being recorded is considered an important function of the system.

In the data reduction phase, one, two or three data channels may be arbitrarily chosen using the channel selectors. The fourth discriminator is used in conjunction with the tape speed compensator. The graphic recorder and the digitizer are both fed directly from the discriminator outputs, which are faithful reproductions of the original data.

The paper tape punch is driven by a $3600 \mathrm{rpm}$ synchronous motor and contact closures operated by the motor provide timing signals and synchronization to the digitizer. The digitizer accepts a maximum of three data channels and digitizes them sequentially. Each channel is switched in, a sample is taken, converted to a seven level binary number and punched out on the paper tape, all in $1 / 60$ second. The same sequence of events follows for the next two channels, a total of $1 / 20$ of a second being required for the complete operation on all three 


\section{AN INSTRUMENTATION SYSTEM FOR WAVE}

MEASUREMENTS, RECORDING AND ANALYSIS

channels. Following this sequence the paper tape is advanced with no punch, thus providing a space, i.e., a fourth channel with zero punch, to separate this data group from the next data group to be punched. Preselected sample rates of $15,10,5,3,2,1$ per second are provided. A time mark generator controlled by the digitizer provides a tick mark on the graphic record at each time a group is sampled.

A seven level binary code was selected for the digital format for two reasons. First, the seven level binary code provides for any number from 0 to 127 thus permitting a digitizing accuracy of better than $1 \%$. The second reason is that with the straight binary code the Teletype tape punch can punch any number up to 127 in a single operation, thus permitting a rapid digitizing rate and also the optimum packing density of information on the paper tape. The relatively fast operation approximates simultaneous digitization of the three data channels if their signals do not vary rapidly. This scanning rate was a specific requirement in setting forth the specifications for the digitizer and tape punch. In any event the time difference between adjacent channels is $1 / 60$ second plus or minus approximately $3 / 4$ millisecond on the average.

There is one disadvantage to the use of the seven level binary code which should be clearly emphasized. No electronic computer known to the authors or their associates uses this particular code for normal entry of data. However, data entry has been easily accomplished in two cases using available paper tape readers. The IBM 46 tape-to-card punch has been used to transfer the data to IBM cards. This IBM 46 has been more effectively used, however, as a tape reader for direct entry of the data into an IBM 650 computer. A tape reader has also been provided for entry of the data directly into the Autonetics Recomp II computer. In both of these computers, through programming, the data is then put into proper format for subsequent calculation. Mr. R. G. Stevens of the WHOI has done all the work pertaining to the entry of the punched paper tape data into these two computers.

The digitizer was specially built because of its particular operating characteristics. The only other piece of equipment which required any special attention in its selection was the tape recorder. Because of the recording of FM signals, in selecting the tape recorder it was necessary to specify that the intermodulation distortion be kept as low as practicable. This was satisfied with the installation of a higher quality recording-reproducing head than normally supplied. However, in so doing, the frequency response of the recorder was reduced to $15 \mathrm{kc} / \mathrm{s}$ at 15 inches/second. It is for this reason that the total number of data channels that could be recorded on each track of the tape was limited to the first twelve, the thirteenth channel being required for tape speed compensation. The recorder has three standard operating speeds, $15,71 / 2$ and $33 / 4$ inches/second, and with a small modification $17 / 8$ inches/second. At this lowest tape speed using standard 1.5 mil magnetic 


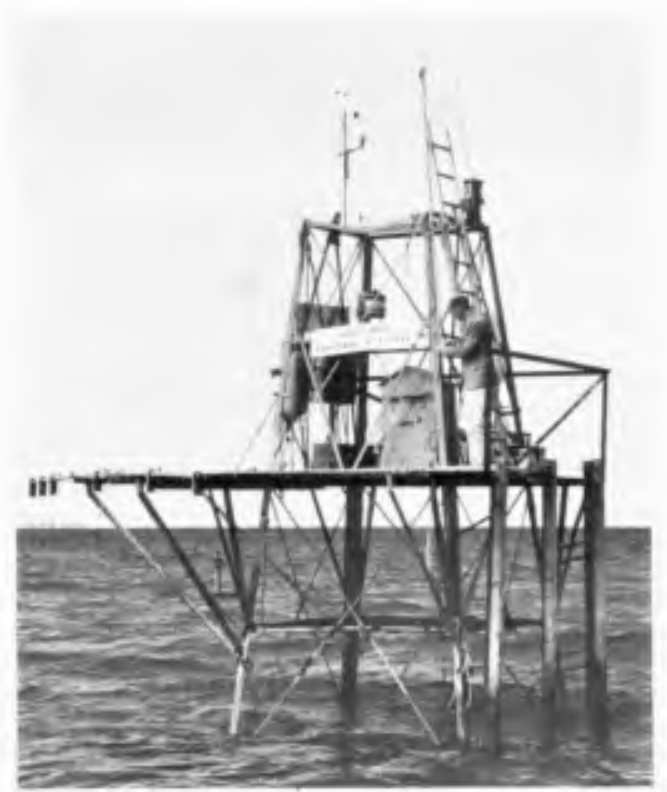

Fig. 6. WHOI tower situated in Buzzards Bay, Mass., water depth 42 feet. 0.015 inch diameter SS wire detectors hang from coaxial cables supported off left edge of tower.

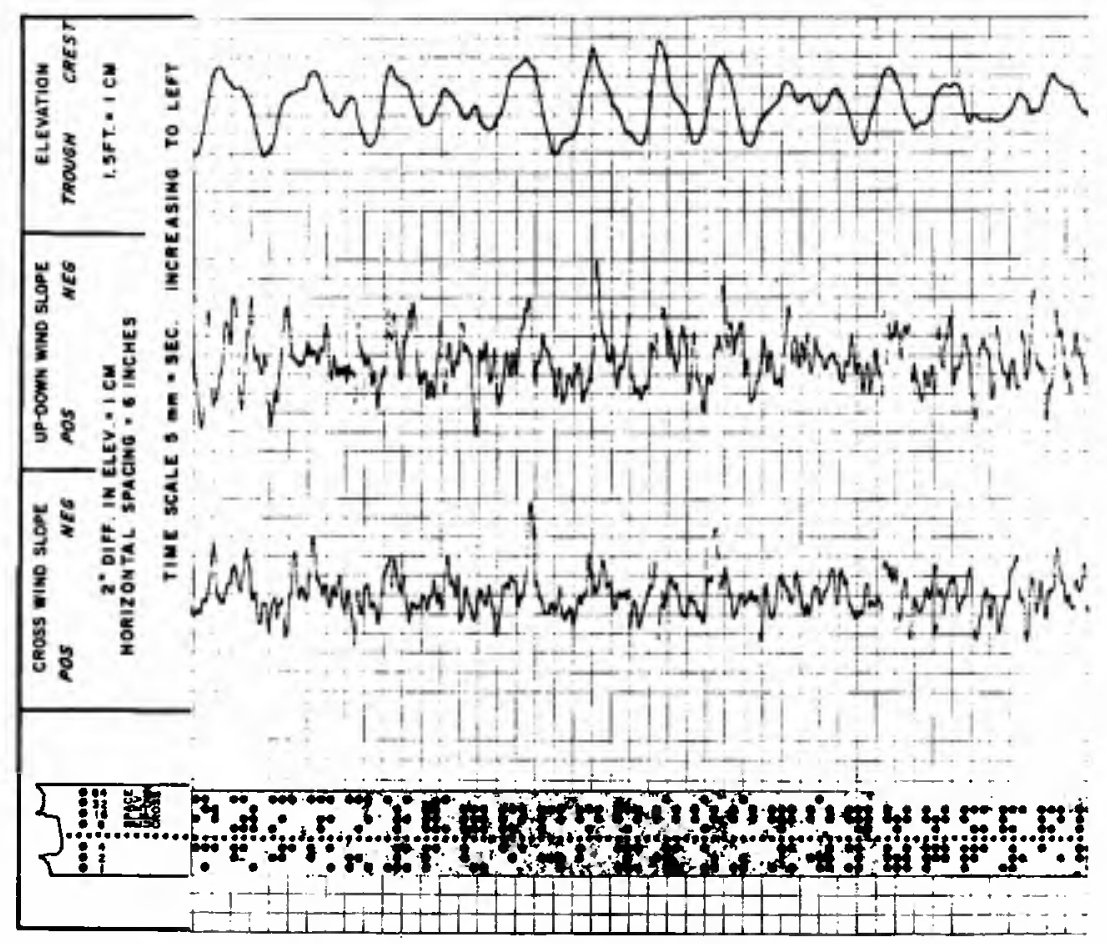

Fig. 7. Sample data obtained with resistance wire detectors. The heave lines are at $5 \mathrm{~mm}$ spacing. Bottom figure is sample of punched paper tape digitized at 10 groups per second. Tape is unrelated to data above. 


\section{AN INSTRUMENTATION SYSTEM FOR WAVE \\ MEASUREMENTS, RECORDING AND ANALYSIS}

tape, recordings of eight hours in duration can be made using the first five data channels. By in large, the intelligence frequencies present in oceanographic data are very low. For the wave studies carried out at WHOI it was necessary, however, to be able to record faithfully frequencies up to $10 \mathrm{cps}$. As is apparent in Table $I$, the system can record intelligence frequencies up to $110 \mathrm{cps}$. Thus, with faster digitizing equipment, the system may have a much wider range of application in other types of research. For the WHOI or similar type system further applications more directly related to the field of oceanography have been discussed briefly in Ref. 10 .

In this section the intent has been to describe the data acquisition and reduction system through a rather general description, but of sufficient detail that its operating characteristics are clearly understood. Several practical features of the system have been emphasized because of their versatile and practical aspects. Finally, it is believed that the system presents a unified and logical concept in data handling.

\section{THE FIELD INSTALLATION AND RECENT OBSERVATIONS}

An extensive program of wave observations has been carried out using the equipment described in the preceding sections. A tower was placed in Buzzards Bay in water 42 feet deep and at a location 2 miles line of sight distance from the laboratory in Woods Hole. The tower, which is of light steel frame construction and 60 feet in height, was originally designed as a wind mill structure. The bottom of each leg is fitted with a large box shaped foot to provide a bearing surface. The lower sections of the tower were reinforced so as to support an additional 6 ton weight to "anchor" the tower to the bottom. A large cast concrete and scrap iron anchor had been previously placed at the proper site. The tower is placed over the anchor and attached to it with an hydraulic cylinder. By pumping oil into the cylinder the tower is anchored to the bottom with a predetermined part of the anchor weight. This manner of installation has proven most satisfactory and the tower has withstood winds in excess of 60 knots and waves up to 6 feet in height. The tower is disconnected from its anchor, picked up and carried to shore and stored during the winter months when heavy icing conditions may prevail. A detailed description of this facility is given by Carver, (1958), and an illustration of the tower is given in Fig. 6 .

The wave wires are electrically connected to the input of the meas uring circuits with a relatively inexpensive coaxial cable, RG-58AU. Equal lengths of coaxial cable were used for each wave wire detector. The simplest, most expedient and satisfactory means for holding the resistance wire in place was to hang each wire by means of a clamp on the coaxial cable with a small lead ball of about 3 pounds weight tied 


\section{COASTAL ENGINEERING}

on the end of the wire. The submerged length of wire was about 20 feet so that the small lead ball was not subjected to any appreciable fluid velocities resulting from the wave field. The estimated critical length of the 0.015 inch stainless steel wire was about 3.5 to 4 feet. At no time were the wires observed to move because of wave motion or wind.

Referring to Fig. 6 there may be seen the wooden box in the shape of a dog house which encloses all the necessary electronics for obtaining the wave and wind data and telemetering it to the laboratory. Mounted on masts above the tower are the anemometer and wind direction indicator and the $230 \mathrm{mc}$ tuned ground plane antenna. At the laboratory a corner reflector and antenna were mounted above the roof from which sufficient signal strength was obtained for reliable operation. The accumulator and oil tank for the hydraulic anchoring system are also visible.

On another occasion these same type resistance wires were used from a much larger tower installation. Approximately 40 foot lengths of 0.015 inch resistance wire were used and approximately 25 to 30 feet of this was below water level. Satisfactory wave records were obtained in winds in excess of 40 knots and waves 20 to 25 feet in height. The wires were observed to be in some motion dependent upon the size waves encountered. The maximum horizontal motion of the wire was about 1 foot for the highest and longest waves observed.

A sample record of elevation, up-down wind slope and crosswind slope is shown in Fig. 7. The wind speed at the time of recording was 16 knots, having been this speed for the previous hour and before that time the wind had been blowing at about 10 knots. For the up-down wind slope record the negative sign refers to the slope found, on the average, on the side of the wave facing the direction in which it is propagating. The same rule holds for the crosswind slopes if the wave were traveiing from left to right when looking into the wind. To obtain the actual slope it is necessary to divide the indicated differences in elevation by the wire spacing which was 6 inches.

At the bottom of Fig. 7 there is reproduced a sample of the punched paper tape for a record similar to that above it. The four channels, crosswind slope, up-down wind slope, elevation and space are indicated in the figure. The group punching rate was 10 times/second.

\section{ACKNOWLEDGMENTS}

The developments reported in this paper were made possible through a grant to the Woods Hole Oceanographic Institution from the National Science Foundation of the National Academy of Sciences and in part through a contract with the U. S. Navy Office of Naval Research. 


\section{AN INSTRUMENTATION SYSTEM FOR WAVE MEASUREMENTS, RECORDING AND ANALYSIS}

\section{REFERENCES}

1. Caldwe11, J. M. (1948). An ocean wave measuring instrument: Tech. Memo. No. 6, U. S. Army Corps of Engineers, Washington, D. C.

2. Carver, C. E. Jr. (1958). The WHOI wave tower research facility: Ref. No. 58-51, Woods Hole Oceanographic Institution, Woods Hole, Mass. (Unpublished manuscript)

3. Churchill, R. V. (1948). Introduction to complex variables and applications: McGraw-Hill Book Co., New York

4. Clayton, L., Ivey, H. D. and Teegardin, H. H. (1954). Preliminary study for the development of a sea state meter: Tech. Report No. 6 Engineering Experiment Station Proj. No. 157-96, Georgia Inst. of Tech.

5. Gerhardt, J. R., Jehn, K. H. and Katz, D. (1955). A comparison of step-, pressure- and continuous wire-gage wave recordings in the Golden Gate Channel: Trans. Amer. Geophys. Union Vol. 36, No. 2

6. Guillemin, E. A. (1935). Communication networks: John wiley and Sons, Inc., New York, Vol. II

7. Nichols, M. H. and Rauch, L. L. (1957). Radio telemetry: John Wiley and Sons, Inc., New York

8. Tucker, M. J. and Charnock, H. (1955). A capacitance-wire recorder for small waves: Proc. 5 th Conf. Coastal Eng. Council Wave Res. Univ. of Calif. Berkeley

9. Whittenbury, C. G. (1956). A capacitance probe for recording water waves: Report No. R-84, Control Systems Lab. Univ. of I11. Urbana, I11.

10. Proceedings of the conference on automatic data handling for oceanographic observations (1959) Ref. No. 60 10, Woods Hole oceanographic Institution, Woods Hole, Mass. (Unpublished manuscript) 